\title{
Remesas y crecimiento económico regional en Colombia 2009-2016: una aplicación de datos panel
}

\author{
Remittances and Regional Economic Growth in \\ Colombia 2009-2016: A Panel Data Application
}

\author{
Remessas e crescimento econômico \\ regional na Colombia 2009-2016: uma \\ aplicação de dados em painel
}

Andrés Fernando Chamorro-García*
Edwin Arbey Hernández-Garcia ${ }^{*}$

Fecha de recepción: 7 de noviembre de 2018

Fecha de aceptación: 19 de junio de 2019

Cómo citar este artículo/ To reference this article / Comment citer cet article / Para citar este artigo:

Chamorro-García, A. \& Hernández-García, E. (2019). Remesas y crecimiento económico regional en Colombia 2009-2016: una aplicación de datos panel. Apuntes del CENES, 38(68), 185 - 246. https://doi.org/10.19053/01203053. v38.n68.2019.8576

* Economista. Universidad del Valle, Cali, Colombia. andres.fernando.chamorro@correounivalle.edu.co (3) https:// orcid.org/0000-0002-3295-0209

** Economista. Magíster. Docente e Investigador en el Departamento de Ciencias Sociales de la Universidad Nacional de Colombia, Palmira. Asesor de modelos estadísticos y econométricos, Universidad Cooperativa de Colombia, Cali. edwinarbeyh@gmail.com $@$ https://orcid.org/0000-0002-5919-7659 


\section{Resumen}

El objetivo principal del artículo es determinar el impacto de las remesas sobre el crecimiento económico de las regiones en Colombia, teniendo en cuenta la estructura de datos panel 2009-2016. Se usan los datos de ingresos por remesas internacionales del Banco de la República y el producto interno bruto de las regiones colombianas publicado por el Departamento Administrativo Nacional de Estadística (DANE), a partir de los cuales se evalúan ecuaciones econométricas mediante el estimador de efectos fijos corregidos por autocorrelación y heterocedasticidad para datos panel. Los resultados muestran un efecto positivo y significativo de las remesas en el crecimiento económico regional.

Palabras clave: remesas, crecimiento económico, economía regional, datos de panel.

Clasificación JEL: C33, F22, F24, F43, R11. 


\begin{abstract}
The main objective of the article is to determine the impact of remittances on the economic growth of the regions in Colombia, taking into account the panel data structure for the period 2009-2016. We use the data of international remittance income from the Banco de la República and the Gross Domestic Product of the Colombian regions published by the Departamento Administrativo Nacional de Estadística (DANE), from which econometric equations are assessed using the estimator of fixed effects corrected by autocorrelation and heteroskedasticity for panel data. The results show a positive and significant effect of remittances on regional economic growth.
\end{abstract}

Keywords: remittances, economic growth, regional economics, panel data. 


\section{Resumo}

O principal objetivo do artigo é determinar o impacto das remessas sobre o crescimento econômico das regiões da Colômbia, levando em conta o painel de estrutura de dados 2009-2016. São utilizados os dados de renda das remessas internacionais do Banco da República e do produto interno bruto das regiões colombianas, publicados pelo Departamento Administrativo Nacional de Estatística (DANE), a partir dos quais as equações econométricas são avaliadas por meio do estimador de efeitos fixos. corrigido por autocorrelação e heterocedasticidade para dados em painel. Os resultados mostram um efeito positivo e significativo das remessas no crescimento econômico regional.

Palavras-chave: remessas, crescimento econômico, economia regional, dados em painel. 


\section{INTRODUCCIÓN}

Se considera que las remesas son un fenómeno que se originó a finales de los noventa del siglo anterior, que se explica por el proceso migratorio en diferentes economías del mundo (Leal, 2008). En Colombia, las problemáticas internas que vivía el país, como es el caso de la inseguridad e inestabilidad económica a finales del siglo XX, generaron un gran proceso migratorio por parte de los colombianos que buscaban ayudar a sus familias (Sánchez \& Echeverry, 2014; Urrutia, 2005).

Así mismo, las remesas hacen parte de una temática interesante y que ha tomado fuerza en los últimos años por la gran entrada de capitales que representan, debido al aumento de transferencias de los trabajadores emigrantes desde el exterior (Bohórquez, 2015). Este auge de emigraciones y movimientos de capitales entre los países ha sido tan importante que ha llamado la atención de instituciones como el Banco Interamericano de Desarrollo, que pretenden conocer los efectos que esto puede traer a los países que se benefician de la entrada de dichos flujos de dinero y la mejor manera de aprovechar estos recursos.

Por otro lado, las remesas resultan ser muy importantes para algunos países como México, Guayana y Haití. En el año 2004 , las remesas fueron alrededor del $50 \%$ del producto interno de Haití y cerca del $20 \%$ en Jamaica, Honduras y el Salvador; México, por su parte, fue el país con el mayor flujo de remesas, siendo de esta manera una de las naciones más beneficiadas por estas transferencias (Acosta, Calderón, Fajnzylber \& López, 2008).

Con respecto a Colombia, para el censo del 2005 aproximadamente el $55 \%$ de la emigración estaba representada por el Valle del Cauca con un $23 \%$, Cundinamarca-Bogotá con un $19 \%$ y Antioquia con un $13 \%$. Así mismo, en el nivel regional, el departamento del Valle del Cauca se caracterizaba como un nodo expulsor, debido a que el $6.4 \%$ de los hogares mencionaron tener un familiar emigrante, en comparación con 
el $2.8 \%$ del promedio nacional, Bogotá con un $2.8 \%$ y Antioquia con un $2.7 \%$ (Valencia, 2012).

De acuerdo con Bonilla (2017), las estadísticas presentadas por el Banco de la República entre los años 2005 al 2015 sugieren que al país ingresaron remesas por un valor aproximado de 4149 millones de dólares promedio anual, y que este valor equivale al $1.4 \%$ del producto interno bruto (PIB) del país para el año 2015 , o al $96 \%$ del recaudo anual promedio por regalías entre el 2013 al 2014.

Teniendo en cuenta las anteriores consideraciones, la pregunta de investigación que guía este trabajo es ¿cuál es el impacto de las remesas internacionales sobre el crecimiento económico de las diferentes regiones de Colombia entre los años 2009 a 2016? Por lo tanto, el objetivo general es estimar el impacto de las remesas sobre el crecimiento económico de cada una de las regiones del país que más reciben estos flujos de dinero.

El artículo se divide en seis secciones; en primer lugar está esta introducción. En segundo lugar se hace una revisión de la literatura tanto internacional como nacional sobre el tema de estudio. En la tercera sección se presentan los hechos estilizados de la migración, las remesas y el PIB por departamentos. Luego se comenta la metodología empírica relacionada con las fuentes de información, las variables y la ecuación economé- trica. En quinto lugar se analizan los resultados econométricos. Finalmente se exponen las conclusiones.

\section{MARCO DE REFERENCIAS}

Las remesas son un tema que ha venido despertando interés desde hace varios años por el gran flujo de dinero que representan para los países en desarrollo y la relación que tienen con el aumento migratorio en cada uno de los países. Por esto se han llevado a cabo diversos estudios en relación con este tema, para analizar la influencia de tales recursos en la economía de los países receptores. A continuación se presenta la literatura internacional y luego la nacional.

\section{Revisión de la literatura internacional}

Mendoza y Calderón (2006) analizan el efecto de nuevas características en las tendencias del crecimiento económico mexicano, después de haber transcurrido varios años de la entrada en vigor del Tratado de Libre Comercio de América del Norte (TLCAN), abordando principalmente el impacto de las remesas y las variables de apertura en el crecimiento de los estados en México. Los autores encuentran que las remesas no son significativas para explicar el crecimiento económico, por lo cual no es clara la relación entre esos flujos de dinero y cómo pueden influir en el crecimiento económico regional mexicano. Además, los coeficientes de apertura sugieren que hay un impacto positivo de 
las exportaciones y el comercio regional en el crecimiento.

Por su parte, Acosta, Calderón, Fajnzylber y López (2008) muestran las diferencias entre los países sobre el impacto de las remesas en el desarrollo económico y proporcionan información que ayude a los diferentes gobiernos a utilizar estas transferencias de la mejor manera posible. Con este fin, relacionan estudios de diferentes países que han empleado datos de encuestas de hogares con información de las remesas, todo esto para una gran variedad de países de América Latina. Concluyen que las remesas son importantes para América Latina, pues influyen en el desarrollo económico reduciendo la pobreza $y$ la desigualdad, y proponen que las autoridades por medio de mecanismos pueden mejor el uso de estos recursos influyendo así en el desarrollo.

También, estudios como los de Solimano y Allendes (2007) en colaboración con el Banco Interamericano de Desarrollo, sugieren que las disparidades en el nivel de desarrollo de los países generan el aumento de la migración y que es de gran importancia ser más eficientes en el mercado de remesas por los altos costos de envío, para así tener mejores efectos en el desarrollo económico.

Por su parte, Pradhan, Upadhyay y Upadhyaya (2008) examinan el efecto que tienen las remesas en el crecimiento económico de 39 países en desarrollo.
Para esto utilizan un modelo de crecimiento estándar basado en efectos fijos y aleatorios, en donde el crecimiento del PIB per cápita depende de las remesas, la inversión, la apertura económica y la política. Los resultados sugieren que las remesas tienen un impacto importante en el crecimiento y las otras variables mostraron ser significativas; aunque en el caso de la política se obtienen diferentes resultados dependiendo de la forma en que esta variable se incluye en el modelo empírico, afectando el coeficiente de remesas.

Desde una perspectiva de país, Ochoa (2010) muestra el gran flujo migratorio que vivió Ecuador a finales del siglo $\mathrm{XX}$ a causa de la crisis financiera que se generó en dicha nación y sus efectos, que llevaron a grandes volúmenes migratorios $\mathrm{y}$, por lo tanto, de mayores flujos de dinero hacia el Ecuador. Así mismo, el autor evidencia la relación de las remesas y los flujos migratorios con el crecimiento y el desarrollo regional. $\mathrm{Su}$ conclusión es que las regiones más pobres no presentan los mayores niveles migratorios, mientras que las regiones más pobladas y con mayor crecimiento son las que tienen mayor flujo de emigrantes y, por lo tanto, mayores flujos de ingresos por remesas.

Igualmente, Carvajal y Almonte (2011) analizan por medio de las remesas los efectos que tuvo la crisis económica de los Estados Unidos en diferentes variables económicas en México, como es el caso del nivel de desempleo, el consu- 
mo, la inversión privada y el crecimiento, empleando para esto un modelo de ecuaciones simultáneas. Ellos plantean que la crisis en Estados Unidos afectó el envío de remesas a México, presentándose en el nivel de consumo privado una reducción a corto plazo. Suponen los autores que las remesas pueden manejarse como un elemento del desarrollo y que se puede generar una disminución de la inversión privada y de la tasa de crecimiento de la economía mexicana. Concluyen que se cumple la hipótesis de la nueva economía de la migración laboral, según la cual las remesas permiten el desarrollo económico de México.

El trabajo anterior proporciona una visión diferente a lo mostrado por Mendoza y Calderón (2006), en el sentido de que se evidencia la importancia de las remesas para las familias que reciben estos recursos y para el crecimiento de la economía mexicana, asunto que los primeros no podían identificar.

Por último, Mayoral y Proaño (2015) investigan el efecto de las remesas internacionales en el desarrollo económico de las diferentes naciones de América Latina entre los años 1975-2012, empleando datos de panel, con el fin de encontrar cómo la crisis económica del 2008 influyó en la generación de remesas y en el crecimiento del PIB. Los resultados sugieren que las remesas no han influido directamente en el crecimiento de la gran mayoría de países de América Latina, excluyendo a República Dominicana, El Salvador, Costa
Rica, entre otros, a los cuales les ayudó a conseguir una mayor renta per cápita. Pero después de la crisis del 2008 se producen efectos significativos para los diferentes países de América Latina y el Caribe, impactando positivamente el ingreso per cápita.

\section{Revisión de la literatura nacional}

Con base en los artículos académicos consultados para el caso de Colombia, se evidencia que el tema de las remesas y su impacto en el crecimiento económico regional del país, ha sido poco investigado. Los artículos que estuvieron centrados específicamente en esta cuestión son escasos; se encontró la investigación de Monroy (2010) que trata este tema con el enfoque que se pretende en este trabajo, pero que se diferencia por el periodo de estudio, lo cual muestra una gran diferencia en los resultados, ya que en Monroy las remesas no tienen impactos significativos en el periodo 1994-2007, mientras que en nuestro trabajo se podrá observar que para un periodo más reciente, 2009-2016, con mayores volúmenes de emigración y envío de remesas sí hay un efecto positivo y significativo sobre el crecimiento económico de las regiones. En este mismo sentido, el trabajo de Mayoral y Proaño (2015) muestra que las remesas sí lograron tener un efecto positivo y significativo en la región de América Latina después del año 2008. 
Por su parte, Urrutia (2005) señala la importancia de las remesas internacionales enviadas por las personas que han emigrado a otros países y analiza la evolución de estas transferencias para el caso colombiano. Las conclusiones a las que llega es que Latinoamérica y el Caribe son los principales receptores de remesas familiares; en Colombia se observa una relación importante entre las entradas de estos dineros y el crecimiento del PIB per cápita del país, como también mayores costos al enviar giros desde Estados Unidos a Colombia.

Más adelante, Sinisterra (2005) analiza las principales causas y efectos económicos de la migración laboral internacional. Con base en la teoría del comercio internacional indica cómo un mayor flujo de emigrantes puede aportar al crecimiento económico, tanto en el país de destino como para el país de origen. En el caso del país de destino, al incrementar el factor trabajo que emplea intensivamente en el producto que exporta (efecto Rybczynski), mientras que en el segundo se incentiva el crecimiento al aumentar el ingreso. De ahí el consumo de las familias que reciben las remesas, como también el aumento de la inversión productiva.

Khoudour (2007) analiza minuciosamente los efectos que han tenido las transferencias de dinero tanto en el aspecto económico como social, y también hace énfasis en los riesgos potenciales a largo plazo de estos flujos de dinero. Se concluye que se está consi- derando la emigración como una opción de política pública en Colombia, siendo una manera por parte del Estado de eludir sus responsabilidades, al pasar los problemas internos a otros países, pues la emigración genera una reducción del desempleo y esto se debe solucionar internamente sin pasarle el problema a otros. El autor encuentra que las remesas estimulan la actividad económica.

Por otra parte, Leal (2008) realiza un estudio para el caso colombiano entre 1994 y 2006, con el objetivo de determinar si los envíos de remesas por parte de los emigrantes son por motivos altruistas o por razones de inversión. La conducta altruista es relacionada con una relación contracíclica al hacer las estimaciones de las variables remesas y PIB, debido a que una contracción en el crecimiento sería compensada por un aumento en el flujo de remesas para suavizar el consumo de las familias. También pretende conocer los impactos que tienen estos flujos de dinero en el desarrollo económico del país y realiza varias estimaciones empleando el método de vectores autorregresivos (VAR).

Los resultados sugieren que las remesas internacionales proceden de manera contracíclica a la tendencia del PIB y del consumo, y de forma procíclica con respecto a la inversión del país. Igualmente muestran que los individuos envían remesas por razones altruistas y por inversión, y que los impactos de las remesas en el consumo y el PIB no son muy claros. 
En el ámbito regional, Monroy (2010) analiza el impacto que tienen las remesas sobre el crecimiento de nueve departamentos, los cuales recibieron la mayor cantidad de estos flujos de dinero para el periodo comprendido entre 1994-2007. En esta investigación utilizó datos de panel para estimar el impacto de las remesas en las diferentes regiones del país, con fuentes de información como el Departamento Administrativo Nacional de Estadística (DANE) y el Banco de la República. Aunque la autora comenta, en teoría, que las remesas suelen ser importantes para las familias receptoras, su impacto no es significativo para el crecimiento económico de la región. Según los datos, concluye que el departamento con mayor flujo de remesas es el Valle del Cauca y la variable población influye considerablemente en el orden regional en el crecimiento económico.

La investigación de Monroy (2010) es la que más se relaciona con el tema y la metodología que se pretende estudiar en este artículo. No obstante, en el presente documento se analiza una ventana de tiempo más reciente, 2009-2016, y un grupo mayor de departamentos, 20, además de incluirse otras variables de control como el capital humano, las tecnologías de la información y las comunicaciones, el grado de apertura económica y el comportamiento empresarial de cada departamento. Adicionalmente, se dan a conocer los hechos estilizados de las remesas internacionales luego de la crisis económica mundial del año
2008, los cuales no se analizaron en Monroy (2010).

En el mismo año, Romero y Salinas (2010) examinan los efectos que generó la emigración a finales de los años noventa, por medio de las transferencias en la participación laboral de las familias que reciben estos recursos en el Área Metropolitana Centro Occidente de la zona cafetera colombiana; para esta investigación se empleó una encuesta realizada en esta zona. Los resultados sugieren que la crisis del 2008 ocasionó la disminución del flujo de remesas en algunos países, entre ellos Colombia, influyendo en el mercado laboral colombiano, porque en años posteriores se elevó la participación laboral y el desempleo en esta parte del país. Así mismo, se encuentra que las personas que reciben estas transferencias poco buscan entrar al mercado laboral en comparación con las que no reciben estos dineros.

Otros estudios, como el de Montoya, López, Arboleda, Ramírez y Viana (2010), describen cómo se relacionan las remesas con el crecimiento de la economía colombiana, basados en el sector económico de la construcción. En la investigación de Montoya et al. (2010) se concluye que el altruismo y la inversión son las principales causas de la generación de remesas; el primero tiene una tendencia anticíclica de la economía del país que recibe estos recursos, mientras que la inversión se comporta procíclicamente. Adicionalmente, debido a la gran 
influencia del sector de la construcción en el país, se ha pretendido atraer estos recursos a este sector, para que ayuden a mejorar indicadores sociales como el desempleo y la pobreza.

En el estudio de Valencia (2012) donde se analizan las incidencias de la emigración en la participación laboral de los hogares del área metropolitana de Cali-Yumbo, empleando para esto la Gran Encuesta Integrada de Hogares y un modelo logit basado en la tradición neoclásica de la teoría del consumidor-, se concluye que las remesas influyen significativamente al reducir la posibilidad de participar en el mercado laboral, es decir, se presenta una dependencia económica de los hogares con respecto a las remesas, además las personas que más reciben estos dineros son mujeres cabeza de familia.

Del mismo modo, Sánchez y Echeverry (2014) identifican cómo influyen las remesas en la economía de Colombia, observando el impacto que generan en variables importantes al tomar decisiones de política macroeconómica, como los cambios en el PIB, la creación de tendencias inflacionarias y cambios en la tasa de cambio. La información utilizada es del Ministerio de Relaciones Exteriores de Colombia y del Banco de la República. Se encuentra que las regiones que reciben las remesas están asociadas a flujos migratorios históricos; en el ámbito macroeconómico, las remesas presentan cada vez un menor impacto en el PIB, y la monetización de los flujos de dinero que ingresan al país por remesas, no ha afectado considerablemente las políticas de la autoridad monetaria.

Estos diferentes artículos, tanto internacionales como nacionales, muestran que las remesas son recursos muy importantes para diversos países, por los grandes flujos de dinero que estas representan; sin embargo, es necesario profundizar para conocer con más detalle las implicaciones de estos dineros en el crecimiento económico. En el caso de Colombia, solamente el trabajo de Monroy (2010) está orientado a identificar el impacto que tienen las remesas sobre el crecimiento económico de las regiones en el país, lo cual es poco, dada la importancia de este tema, motivo por el cual es relevante la realización de esta investigación para aportar a esta literatura, mayormente después de las crisis financiera internacional de 2008 cuando los flujos migratorios crecieron aún más.

\section{HECHOS ESTILIZADOS}

En las últimas décadas en Colombia se presentaron diferentes periodos en los cuales se dieron grandes migraciones hacia el exterior; la primera ocurrió en los setenta, la segunda en los ochenta y la más reciente en los años noventa, motivada principalmente por la inseguridad y la inestabilidad económica del país de aquel tiempo (Sánchez \& Echeverry, 2014). Estos emigrantes han sido los principales generadores de remesas 
hacia el país, debido a que el objetivo de estas personas es ayudar a sus familias a mejorar la calidad de vida de cada una de estas.

Para el año 2016, Colombia ocupaba el puesto 25 en el número de emigrantes con 2,6 millones y el puesto 30 en el total de remesas recibidas. Aunque no es un país que dependa en gran medida de las remesas, no se trata de una situación a la que se le preste poca atención (Bonilla, 2017). Un rasgo interesante para mencionar es que ciertos departamentos son los que poseen el mayor número de emigrantes en el exterior (Figura 1 Panel A), como es el caso del Valle del Cauca con un $23 \%$ del total de emigrantes colombianos en el exterior, seguido de Cundinamarca y Bogotá con $18 \%$ y Antioquia con $13 \%$, según datos del DANE del censo en el año $2005^{1}$.

Así mismo se observa en la Figura 1 Panel A que el fenómeno de emigración mantuvo una tasa de crecimiento positiva, por ejemplo, para el departamento del Valle del Cauca, antes de 1996 el 5 \% de los emigrantes eran de ese departamento, mientras que entre 1996-2000 la tasa de emigración creció al $7 \%$ y para el periodo 2001-2005 nuevamente creció al $11 \%$, así mismo para la región de Cundinamarca-Bogotá aumentó la emigración del $4 \%$ antes de 1996 al
$9 \%$ entre 2001-2005. Para Colombia, en general, la tasa de emigración pasó antes de 1996 del $28 \%$ al $46 \%$ en el periodo 2001-2005, lo cual evidencia en el país lo que ha sucedido en el resto del mundo, y es la creciente migración que se ha dado entre países, por ejemplo, del informe de la Organización Internacional para las Migraciones escrito por McAuliffe y Ruhs (2018) se calcula que el total de migrantes en el mundo creció en $11 \%$ en el periodo $2000-2005$ y $42 \%$ entre 2000-2015.

Por su parte, en el Panel B de la Figura 1 se observa que los principales países de destino de los colombianos en el exterior según información del Censo General del DANE 2005 fueron Estados Unidos con el $35 \%$, $23 \%$ España y Venezuela con $23 \%$ de emigrantes, lo cual indica que antes del año 2005 la emigración colombiana estaba en un $78 \%$ concentrada en estos tres países. En cuanto a la dinámica de emigración, se puede observar en el Panel B que la tasa de salida creció para países como España, que pasó antes de 1996 del $8 \%$ al $29 \%$ en el periodo 2001-2005; para Ecuador y Canadá, del $2 \%$ y el $1 \%$ antes de 1996 al 4\% y 3\% en 2001-2005, respectivamente; mientras que para países como Estados Unidos y Venezuela, la tasa de emigración bajó del $43 \%$ y $32 \%$ antes de 1996 al $27 \%$ y $17 \%$ entre 2001-2005, respectivamente.

1 Este es el último año de referencia, porque el censo actual del año 2018 aún no tiene publicados los microdatos y, por lo tanto, tampoco el módulo de migraciones. 


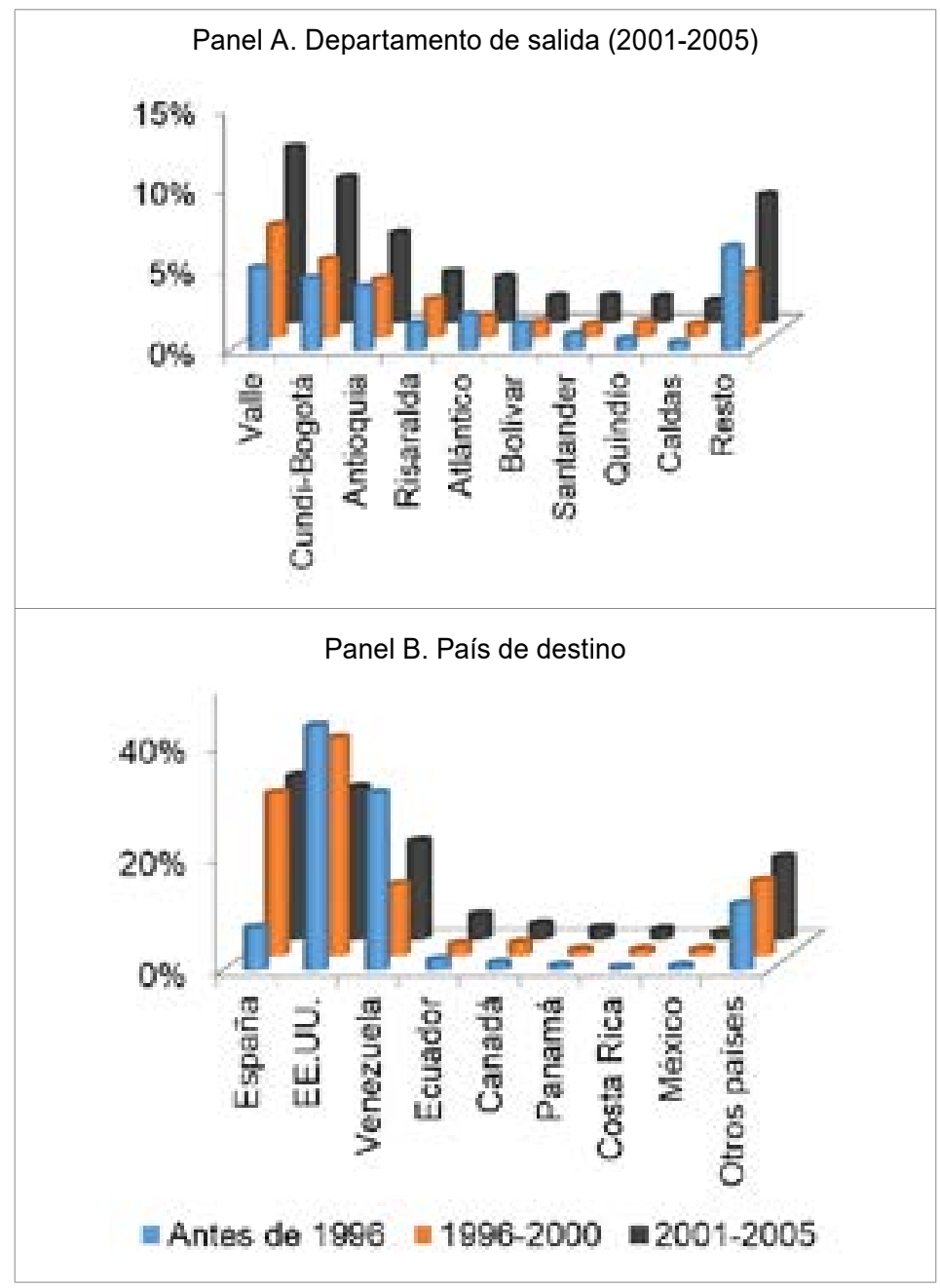

Figura 1. Emigrantes colombianos.

Fuente: cálculos propios con base en datos del DANE (2017) y la CEPAL (2017).

Por otro lado, en la Figura 2 se observa que los ingresos por remesas internacionales han respondido a los choques en las diferentes economías internacionales y variables como la tasa de cambio. Uno de estos es la caída que se aprecia entre el año 2009 y el 2012 con ingresos desde 4829 millones de dólares constantes base 2010 para el año 2009 a 3418 millones de dólares constantes base 2010 en el 2012, lo que se explica por las repercusiones de la crisis financiera internacional en el año 2008, que tuvo origen en los Estados Unidos, país 
desde donde las remesas cayeron en términos reales en $22 \%$, la falta de empleo en España desde donde las remesas cayeron en $50 \%$ y la devaluación del tipo de cambio que pasó de 2156 pesos por dólar en 2009 a 1798 en 2012, con una caída del $17 \%$. No obstante, a partir del año 2013 se presenta una importante recuperación en los ingresos por remesas al país, incrementándose considerablemente desde el año 2014 con ingresos desde 3772 millones de dólares a 6347 millones de dólares en 2016 base 2010 (68\% de crecimiento).
Cuando se analizan las remesas como participación del producto interno bruto (PIB) de Colombia (Figura 2), se encuentra que la participación de las remesas varió en la misma dirección que los ingresos por remesas. Además de estar relacionada la caída en el periodo 2009-2012 con la crisis financiera mundial y que generó incertidumbre por la lenta recuperación de la economía estadounidense, se puede agregar la falta de empleo en España (Sánchez \& Echeverry, 2014), siendo estos los dos principales países donde residían los emigrantes colombianos, como se puede apreciar en la Figura 1.

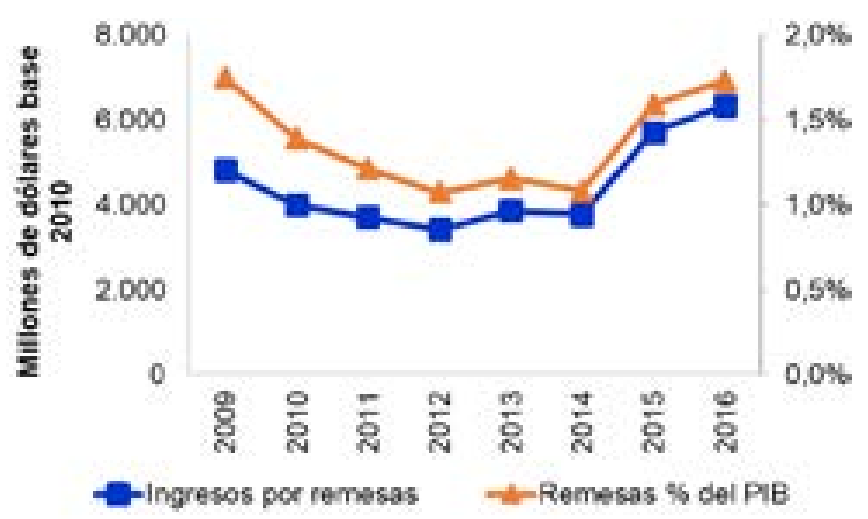

Figura 2. Remesas

Fuente: cálculos propios con base en datos del Banco de la República (2018) y el Banco Mundial (2018).

Además, según se puede observar en la Figura 3 Panel B, gran parte de las remesas que ingresaron a Colombia provenían de los Estados Unidos y España, que, como se mencionó antes, son los principales países de destino de los colombianos. De los Estados Unidos, para el año 2009 llegaban a Colombia 1554 
millones de dólares constantes base 2010, para el año 2012 caían las remesas a 1208 millones de dólares (-22\%), que luego se recuperarían porque para el año 2016 crecerían las remesas al valor de 2907 millones de dólares constantes base 2010 (141 \% respecto al año 2012), lo cual, entre otras razones, se debió a la revaluación del tipo de cambio al pasar de 1798 pesos por dólar en 2012 a 3053 en 2016, con una revaluación acumulada del $70 \%$.

Por su parte, España, segundo país con mayor origen de remesas, redujo su generación entre el año 2009 a 2012 en $50 \%$, pasando de 1961 millones de dólares a 989 millones; sin embargo, entre el año 2012 y el 2016 mejoró levemente (15\%) la producción de remesas, al pasar de 989 millones a 1133 millones de dólares constantes de 2010. A pesar de la recuperación de remesas desde España entre el año 2012 y 2016, comparativamente con el año 2009, este país redujo las remesas en términos reales en un $42 \%$ (Figura 3 Panel C).

En general, estos dos países, Estados Unidos y España, proveyeron remesas por cerca del $67 \%$ para el periodo 20092016, lo que muestra la alta dependencia de Colombia de ellos en términos de ingresos por remesas internacionales. Aunque hay que destacar el hecho de que otros países han tenido más participación en las remesas que llegan a la economía colombiana, especialmente de los latinoamericanos como Chile, Ecuador, Panamá y México, que crecieron en términos reales del año 2009 al año 2016 en $1.089 \%$, $427 \%$, $241 \%$ y $111 \%$, respectivamente (Figura 3 Panel C).

La otra perspectiva interesante que se debe mencionar son los diferentes departamentos receptores de estas transferencias de dinero desde el exterior. En la Figura 3 Panel A se observa que las remesas en el periodo 2009-2016 están concentradas en un $78 \%$ principalmente en cinco departamentos: Valle del Cauca (30\%), Antioquia (17\%), Cundinamarca-Bogotá (16\%), Risaralda (11\%) y Atlántico (5\%). Remesas que, en general, se explican porque la mayor parte de emigrantes residentes en el extranjero salen de estos departamentos. Con base en datos del censo del año 2005, se pude identificar que del Valle del Cauca el destino principal fue España con el $47 \%$ de emigración del departamento, de Cundinamarca-Bogotá el destino principal fue Estados Unidos con el $41 \%$, de Antioquia fue Estados Unidos con el $44 \%$, de Risaralda fue España con el $60 \%$ y del Atlántico fue Venezuela con el $48 \%$, lo que muestra lo mencionado antes, y concentración de emigrantes y remesas en pocos destinos internacionales. 
Panel A. Departamento de destino

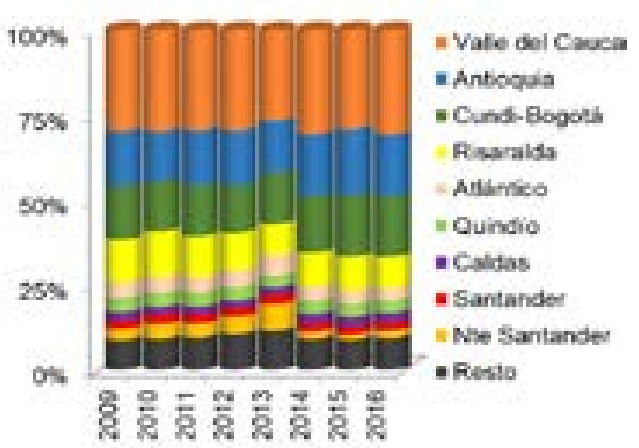

Panel B. País de origen

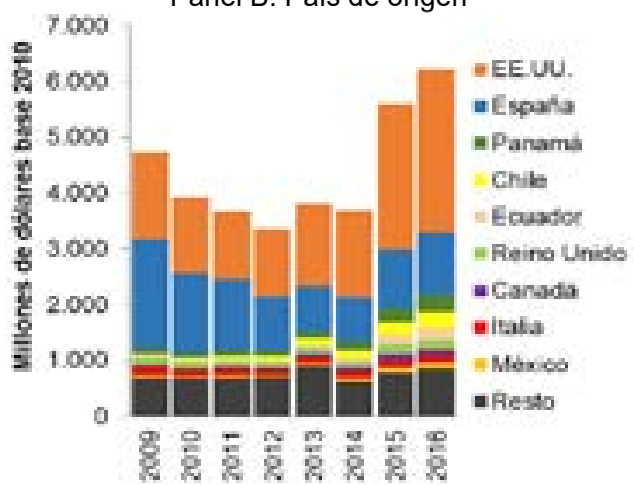

Panel C. Tasa de crecimiento entre 2009 y 2016 por país de origen

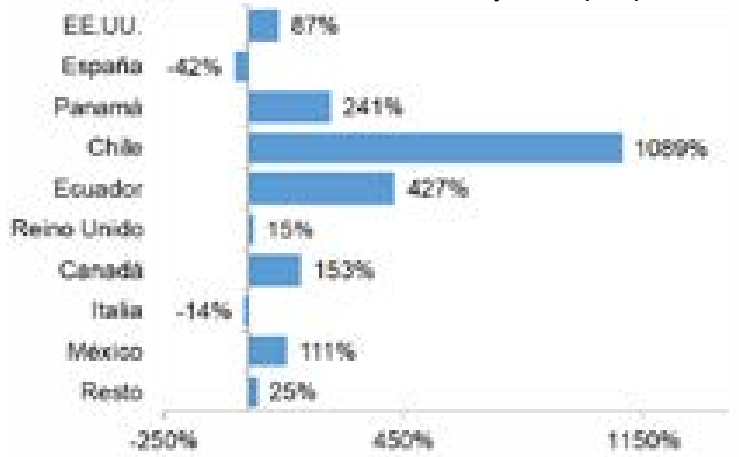

Figura 3. Ingresos de remesas por departamento de destino y país de origen.

Fuente: cálculos propios con base en datos del Banco de la República (2018).

Por último, se puede tener en cuenta que la forma en que están distribuidos los ingresos por departamentos está relacionada con los patrones de emigraciones internacionales (Bonilla, 2017) y, por lo tanto, la generación de remesas también se encuentra conectada con los ingresos en los departamentos. Es así como en la Figura 4 Panel A se observa que el mayor promedio de remesas recibidas se relaciona positivamente con la mayor tasa de emigración, de forma tal que, por ejemplo, el Valle del Cau- ca tuvo un promedio de ingresos por remesas por valor de 1195 millones de dólares entre 2009-2016 y la mayor tasa de emigración del $23 \%$ antes del año 2005, y por el contrario, departamentos con menores tasas de emigración como Boyacá (1 \%), Córdoba (1 \%) y Nariño (1 \%), tuvieron ingresos promedio por 13, 18 y 21 millones de dólares, respectivamente.

Aunque menos pronunciada, también se puede observar la relación positiva 
entre el PIB de cada departamento y los ingresos por remesas internacionales que llegan a cada uno. Así es como, por ejemplo, en los departamentos del Valle del Cauca, Cundinamarca-Bogotá y Antioquia, mayores receptos de remesas, se tiene la mayor producción interna bruta. Este punto es bastante interesante, ya que precisamente es el alcance de este artículo, de donde se pretende mostrar la relación positiva que hay entre los ingresos por remesas internacionales y el crecimiento económico de los departamentos en Colombia. En términos generales, la relación causal se puede explicar desde la demanda, porque a mayores ingresos por remesas, las familias pueden consumir una mayor cantidad de bienes y servicios (Uribe, 2005), y desde la oferta desde la creación de pequeñas unidades de negocios que generan mayor producción y empleo. (Woodruff \& Zenteno, 2001; Alquinga, 2014; Zheng \& Musteen, 2018)

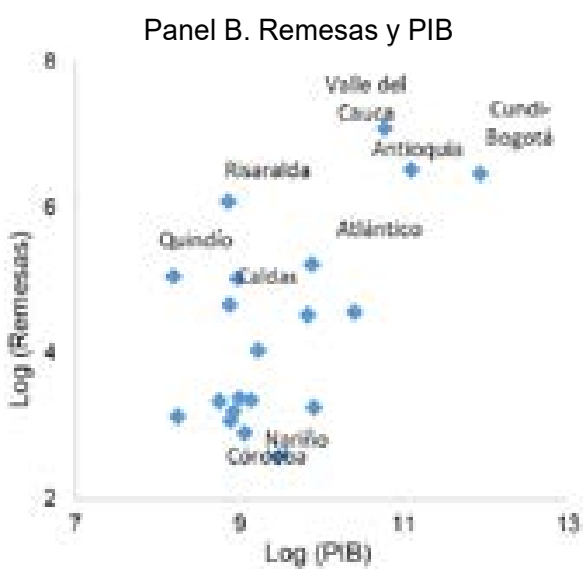

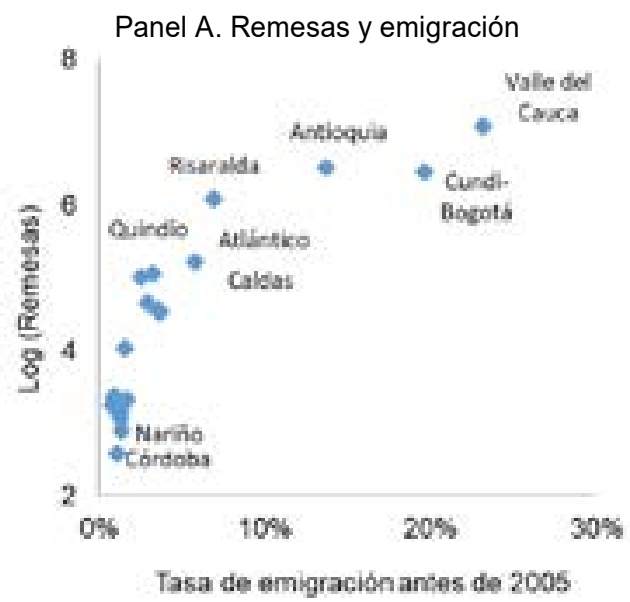

Figura 4. Relación entre las remesas, la tasa de emigración y el PIB (promedio 2009-2016).

Fuente: cálculos propios con datos del Banco de la República (2018) y DANE (2018).

\section{METODOLOGÍA EMPÍRICA}

El análisis empírico relaciona las dos principales variables de interés, el PIB y las remesas por departamentos, pero además se tienen en cuenta otras variables que sirven de controles que explican el crecimiento económico de las regiones. Así mismo, se añade una variable dicotómica que permita controlar el efecto del ciclo económico de las economías extranjeras, principalmente de países como Estados Unidos y España.

La base de datos se empalmó para los periodos 2009-2016 y 2013-2016 con frecuencia anual para los veinte departamentos más representativos y de los cuales hay información completa. Estos 
departamentos son: Valle del Cauca, Cundinamarca-Bogotá, Antioquia, Risaralda, Atlántico, Norte de Santander, Quindío, Caldas, Bolívar, Santander, Tolima, Cesar, Sucre, Huila, Magdalena, Meta, Córdoba, Nariño, Cauca y Boyacá. De esta manera se consolida una estructura de datos panel con ocho periodos y veinte departamentos, para un total de 160 observaciones entre 2009-2016, y de 80 observaciones entre 2013-2016, este último periodo debido a que las variables de control que tienen como fuente de información el Índice Departamental de Competitividad (IDC) empezaron a ser publicadas desde el año 2013.

Dado que la estructura de la información de los departamentos a lo largo del tiempo corresponde a datos panel, en la siguiente ecuación se presenta de manera general la relación entre las variables:

$$
\begin{gathered}
\ln P_{I B_{i t}}=\alpha+\beta \ln \text { Remesas }_{i t}+ \\
\sum_{j=1}^{5} \ln X_{i t} \delta_{j}+\eta_{i}+\mu_{i t}
\end{gathered}
$$

Donde $\ln P I B_{i t}$ es la variable dependiente en logaritmo natural que permite medir el crecimiento económico para el departamento $i$ en el periodo $t$. La variable de remesas está etiquetada como ln Remesas $_{i t}$ en logaritmo natural y hace referencia a la variable independiente con la cual se pretende alcanzar el objetivo del artículo. Esta variable también se encuentra por periodos y los departamentos mencionados antes.
Por su parte, la matriz $X_{i t}$ representa las variables de control que tienen efectos sobre el crecimiento económico para cada departamento $i$ en el periodo $t$. Dentro de esta matriz se consideran cinco grupos de variables independientes, a saber: (i) el capital humano que se relaciona con dos variables específicas, la primera con el número de individuos graduados de educación superior y la segunda es la componente principal de variables relacionadas con el Pilar de Competitividad Departamental conocido como educación y capacitación, (ii) el sector público relacionado con dos variables específicas, la primera que tiene en cuenta el impacto de la inversión pública y la segunda que mide el impacto de la transparencia institucional y administrativa, (iii) el sector externo relacionado con el grado de apertura económica, (iv) las tecnologías de la información y las comunicaciones, y (v) la dinámica empresarial.

Adicionalmente, dentro la matriz $X_{i t}$ se encuentra la variable dummy que permite controlar el efecto del ciclo económico. Esta variable se ha introducido mediante un efecto multiplicativo a las remesas para los años 2009, 2011, 2012, 2013 y 2016, periodos en los cuales las tasas de crecimiento de países como Estados Unidos y España estuvieron a la baja o en escenarios negativos. Se tomaron estos países como referencia, porque de ellos es que proviene la mayor parte de las remesas, como se puede observar en la Figura 3 Panel B. 
En términos generales, con la ecuación (1) y respectivas variables de control se pretende explicar los efectos sobre el crecimiento económico tanto por el lado de la demanda como por el lado de la oferta. Es así como la variable de inversión o gasto público haría parte de la demanda; por su parte, las variables de capital humano, la transparencia institucional y administrativa en el gobierno regional, y la dinámica empresarial, harían parte de la oferta, mientras que las variables de remesas, grado de apertura económica y las TIC tendrían efectos tanto de demanda como de oferta.

Las variables $\eta_{i}$ y $\mu_{i t}$, son los residuos de la ecuación econométrica. En el caso de $\eta_{i}$ es el efecto no observable específico para cada departamento, invariante en el tiempo. $\mu_{i t}$ es el error estocástico que se distribuye independiente e idénticamente con $\mathrm{E}\left(\mu_{i t}\right)=0 \mathrm{y} \operatorname{Var}\left(\mu_{i t}\right)=\sigma^{2}>0$.

Las fuentes de información fueron: el DANE, el Banco de la República, la Dirección de Impuestos y Aduanas Nacionales (DIAN), el Departamento Nacional de Planeación (DNP), el Ministerio de Hacienda (Min-Hacienda), el Observatorio Laboral de Educación (OLE) del Ministerio de Educación y el Índice Departamental de Competitividad (IDC), siendo este último una fuente de datos anual construida por parte del Consejo Privado de Competitividad y del Centro de Pensamiento en Estrategias Competitivas de la Universidad del Rosario, desde el año 2013. A continuación, la Tabla 1 presenta la distribución de las variables según sus fuentes de información:

Tabla 1. Fuentes de información

\begin{tabular}{|c|c|c|}
\hline \multicolumn{2}{|c|}{ Variables } & $\begin{array}{l}\text { Fuente de } \\
\text { información }\end{array}$ \\
\hline \multicolumn{2}{|c|}{ Producto interno bruto } & DANE \\
\hline \multicolumn{2}{|l|}{ Remesas } & Banco de la República \\
\hline \multirow{2}{*}{$\begin{array}{l}\text { Sector } \\
\text { público }\end{array}$} & Inversión pública & DNP y Min-Hacienda \\
\hline & Transparencia institucional y administrativa & IDC \\
\hline \multirow{2}{*}{$\begin{array}{l}\text { Capital } \\
\text { humano }\end{array}$} & Graduados de educación superior & OLE \\
\hline & Educación y capacitación superior & IDC \\
\hline $\begin{array}{l}\text { Sector } \\
\text { externo }\end{array}$ & $\begin{array}{l}\text { Exportaciones e importaciones, que junto con el PIB se } \\
\text { construye el Índice de Apertura Económica }\end{array}$ & DANE-DIAN \\
\hline \multicolumn{2}{|c|}{ Tecnologías de la información y las comunicaciones } & IDC \\
\hline \multicolumn{2}{|c|}{ Dinámica empresarial } & IDC \\
\hline
\end{tabular}

Fuente: elaboración propia. 
Respecto a los efectos marginales esperados, con relación a los ingresos de remesas internacionales se aguarda un efecto positivo sobre el crecimiento económico regional. Las remesas han sido una fuente de ingresos importante en diversas naciones desde finales del siglo XX por la gran cantidad de capitales que estas representan, situación que no ha sido ajena a Colombia, por el gran flujo de emigrantes que se originó a finales de los años noventa y porque muchas familias se han visto beneficiadas por las transferencias provenientes desde el exterior (Urrutia, 2005). En el contexto colombiano, trabajos como los de Urrutia (2005) y Monroy (2010) comentan la relación positiva entre las remesas y el crecimiento del PIB per cápita del país en el primer caso y en las regiones colombianas en el segundo.

Del impacto del capital humano sobre el crecimiento económico, la literatura es amplia en términos del efecto positivo que tiene la formación en capital humano, especialmente la educación, donde la formación del conocimiento en los individuos en actividades productivas trae consigo mejoras en la forma de hacer las tareas empresariales, así como en la interacción con el uso de las tecnologías de la información que incrementan exponencialmente la producción (Lucas, 1988; Romer, 1990; Barro \& Lee, 2013).

En cuanto al efecto del sector público, se espera que sea positivo sobre el crecimiento económico regional, tanto por el lado de la demanda debido a las compras que llevan a cabo las entidades públicas de los departamentos, como por el lado de la oferta debido al manejo institucional transparente y a la eficiencia administrativa que facilitan los procesos jurídicos y organizacionales de las empresas. Por su parte, la relación entre el índice de apertura económica y el crecimiento económico se espera que sea positiva, entre otras razones porque para las economías es importante el comercio con otros países como un mecanismo para incrementar la eficiencia en la producción, la frontera de posibilidades de producción y de consumo (Torres, 2008; Hernández \& Raffo, 2016; Raffo, Hernández \& Díaz, 2018; Krugman, Obstfeld \& Melitz, 2012).

Igualmente se espera un impacto positivo de la dinámica empresarial sobre el crecimiento económico, principalmente porque una mayor dinámica revela que la confianza de los empresarios es positiva y, por lo tanto, sus expectativas son favorables para llevar a cabo mayores inversiones productivas y negocios, lo cual se traduce en mayor producción y generación de empleos (Delfín \& Acosta, 2016; Cardona, Montenegro \& Hernández, 2017).

Además, el uso de las tecnologías de la información y las comunicaciones (TIC) tiene también un efecto positivo sobre el crecimiento económico, que por el lado de la oferta se relaciona con la mejora en la eficiencia de la producción a través de la automatización, la mayor productividad laboral y la eficiencia adminis- 
trativa, aumentando considerablemente la capacidad de almacenar, organizar y procesar información más rápido y a menor costo; así como por el lado de la demanda, mejorando la eficiencia en tiempos de compras de productos en línea y seguridad en las transacciones (Pavlou, 2003; García \& López, 2012;
Organización para la Cooperación y el Desarrollo Económicos [OCDE], 2017; Cardona et al., 2017).

De esta manera se pueden resumir los efectos marginales esperados para la ecuación de la siguiente manera:

Tabla 2. Resumen de efectos esperados

\begin{tabular}{ccc}
\hline & Variables independientes & $\begin{array}{c}\text { Signo } \\
\text { esperado }\end{array}$ \\
\hline \multirow{2}{*}{ Capital humano } & Remesas & + \\
\cline { 2 - 3 } & Graduados de educación superior & + \\
\hline \multirow{2}{*}{ Sector público } & Transparencia institucional y administrativa & + \\
\cline { 2 - 3 } & Apertura económica & + \\
\hline Sector externo & Inversión pública & + \\
\hline & Tecnologías de la información y las comunicaciones (TIC) & + \\
\hline
\end{tabular}

Fuente: elaboración propia.

\section{RESULTADOS}

Con relación a las estimaciones econométricas, la Tabla 3 muestra las estimaciones de parámetros para la ecuación según la metodología de datos panel mediante efectos fijos. Se presentan cinco modelos, que van desde la regresión simple (modelo 1) hasta el modelo 5 , que se podría decir es el más completo, y que según la variable independiente introducida en la ecuación estimada, pone a prueba la consistencia en el impacto marginal de las remesas sobre el crecimiento económico de cada departamento. Así mismo, en la Tabla 3 se observan los estadísticos de prueba, con el fin de estimar el panel por datos agrupados por efectos fijos o efectos aleatorios.

El procedimiento para la selección de modelos y corrección de supuestos en cada uno de los modelos, se hizo de la siguiente forma: en primer lugar, la prueba de Breusch-Pagan rechazó la hipótesis de estimar por mínimos cuadrados ordinarios datos agrupados y aceptó la estimación por efectos aleatorios para datos panel; luego, mediante la prueba $\mathrm{F}$ 
rechazó la hipótesis de estimar por mínimos cuadrados ordinarios datos agrupados y aceptó la estimación por efectos fijos para datos panel; por último se aplicó la prueba de Hausman para elegir la estimación por efectos aleatorios o efectos fijos, de forma tal que la prueba estuvo a favor del estimador de panel con efectos fijos en los cuatro modelos.

También se aplicaron los test que permitieron conocer si los datos presentaban problemas de heterocedasticidad y autocorrelación. La Tabla 3 muestra cada una de las pruebas. Para los modelos estimados por efectos fijos, la evaluación de supuestos se hizo de la siguiente manera: en el caso de autocorrelación se llevó a cabo el test de Wooldridge, de forma tal que en todos los modelos se aceptó la hipótesis alterna de autocorrelación. En el caso del test de heterocedasticidad se hizo la prueba de Wald, encontrándose la aceptación de la hipótesis alterna de heterocedasticidad. Por lo tanto, siguiendo a Beck y Katz (1995) y Beck (2001) y siendo necesario corregir dichas violaciones al supuesto de esfericidad de las perturbaciones, los modelos se estimaron bajo efectos fijos corregidos por autocorrelación y heterocedasticidad mediante errores estándar (PCSE).

A continuación se interpretan los resultados de las estimaciones por datos panel de efectos fijos corrigiendo autocorrelación y heterocedasticidad. De esta manera, la Tabla 3 muestra que las remesas presentan un parámetro estimado positivo y significativo, es decir, que las remesas influyen en el crecimiento del PIB en el nivel regional, tanto en el modelo de regresión simple (modelo 1) como en los modelos 2 al 5, que tienen en cuenta aquellas variables que explican el crecimiento económico, incluyendo el modelo 3 que considera el ciclo económico de economías extranjeras y, por lo tanto, la variación de las remesas. Este impacto positivo se puede explicar vía demanda, porque parte de los ingresos por remesas se gastan en consumo, pero también vía oferta mediante inversiones en negocios y emprendimientos.

En el caso del gasto en consumo se puede ver desde la demanda por bienes o productos, pero también desde servicios, como por ejemplo la educación. Uribe (2005) y Urrutia (2005) comentan que las remesas se pueden convertir en fuente de crecimiento de la demanda interna por diversos productos de consumo, bienes domésticos, compra de vivienda y servicios como el de salud y la educación; por ejemplo, se da un incremento de servicios educativos que promueven la asistencia a la escuela de los niños y también en la respectiva inversión de una educación con mayor calidad.

Por la vía de la oferta con inversiones en negocios, emprendimientos y microempresas, se observa que los departamentos con mayor destino de las remesas (figuras 3 y 4 ) fueron aquellos con mayor densidad empresarial según 
el informe del Índice Departamental de Competitividad para el año 2016 (CPC, 2016); por ejemplo, Valle del Cauca ocupó el puesto 4 de 26 departamentos, Antioquia el 5, Cundinamarca el $11 \mathrm{y}$ Bogotá el 1, y Atlántico el 2. También Ramírez y Aguas (2015) afirman que Cundinamarca, Bogotá, Antioquia, Caldas, Santander, Risaralda y Valle del Cauca, son los departamentos con las posiciones más altas en el escalafón de competitividad para el año 2015, que a su vez son regiones reconocidas por sus aportes al crecimiento económico de la economía colombiana.

Así mismo, trabajos como los de Woodruff y Zenteno (2001), Alquinga (2014) y Zheng y Musteen (2018) demuestran la correlación positiva que existe entre la generación de negocios productivos con las remesas. Por su parte, Uribe (2005) destaca que las remesas se convierten en fuente importante de capital para las microempresas, y que en países como Brasil y Guatemala el $10 \%$ de las remesas financian la creación de nuevos negocios.

En el caso de las variables de control, se puede observar que: (i) del capital humano el efecto marginal es positivo y significativo. En esta dirección, la literatura del efecto positivo del capital humano sobre el crecimiento económico valida lo encontrado, donde diversas investigaciones teóricas y empíricas demuestran que la preparación, la educación y la capacitación como capital humano y este, a su vez, como factor de producción, generan eficiencias productivas en las empresas y externalidades positivas que impactan el desarrollo económico (Lucas, 1988; Romer, 1990; Mankiw, Romer \& Weil, 1992; Weil, 2006; Barro \& Lee, 2013; Marín, Hernández \& Burbano, 2018).

Con relación a la inversión pública, los modelos 2, 3 y 5 evidencian que el PIB a nivel regional se incrementa cuando aumenta dicha inversión, siendo el resultado positivo y significativo. Esto indica que un aumento en las compras del sector público en los departamentos analizados aporta al crecimiento económico regional, generando el resultado que se esperaba de acuerdo con la teoría keynesiana, de que el gasto público tiene un efecto positivo en el crecimiento (Krugman \& Wells, 2016). Adicionalmente, investigaciones como las de Campo y Mendoza (2018) han mostrado un efecto positivo y significativo del gasto público (funcionamiento e inversión) sobre el PIB, evidenciando que el gasto público aporta al crecimiento económico.

Sin embargo, si se tiene en cuenta el orden institucional relacionado con la transparencia y eficiencia administrativa, se puede observar en los modelos 4 y 5 una contradicción, pero que deja ver un aspecto interesante sobre la injerencia institucional en el crecimiento económico de las regiones en Colombia. Lo primero que hay que decir es que en los dos modelos el impacto de la administración pública no es signifi- 
cativo para explicar el crecimiento; sin embargo, solo en el modelo más completo, el quinto, se observa que el efecto estimado es positivo y corresponde a lo esperado, de manera que en presencia de la inversión fiscal, la administración pública genera un impacto positivo. Dicho de otro modo, solo con presencia del gasto público es que la administración puede tener efectos positivos sobre el desarrollo y el crecimiento de la economía regional en Colombia. Por supuesto, ampliar este resultado se sale del objetivo del artículo y queda para ser ahondado en investigaciones posteriores.

Por otra parte, el índice de apertura económica tiene efectos positivos y significativos sobre el crecimiento económico, mostrando así que en los departamentos de Colombia la relación comercial con otros países es un importante detonador de crecimiento y desarrollo, tanto por la ampliación del mercado para la venta de productos locales y generación de divisas, como de la importación de bienes de capital que sirvan para mejorar los procesos productivos en las empresas de las regiones (Torres, 2008; Hernández \& Raffo, 2016; Raffo, Hernández \& Díaz, 2018; Krugman, Obstfeld \& Melitz, 2012). Además, trabajos como los de Awokuse (2003), Sannassee, See- tanah y Jugessur (2014) y Casas (2015) muestran empíricamente una relación positiva entre las exportaciones y el crecimiento económico.

En cuanto al impacto de las TIC sobre el crecimiento, se puede ver que el impacto es el esperado en los modelos 4 y 5 ; sin embargo, solamente resulta significativo en el modelo más completo que es el 5. Como se dijo antes, esta variable tiene efectos muy importantes sobre el crecimiento, pues por una parte mejora la eficiencia de la producción e incrementa la eficiencia administrativa con la forma de procesos y organiza más rápido la información, y por la otra permite que las compras por internet sean rápidas y cada vez más seguras (Pavlou, 2003; García \& López, 2012; OCDE, 2017; Cardona et al., 2017).

Finalmente, la variable de dinámica empresarial impacta de forma positiva y significativa el crecimiento económico de los departamentos colombianos, lo que relacionado con la oferta económica tiene que ver con la mayor generación de empresas y negocios, así como con el incremento de las expectativas empresariales para mejorar la inversión, incrementar el empleo y la producción local (Delfín \& Acosta, 2016; Cardona et al., 2017). 
Tabla 3. Resultados de las estimaciones

\begin{tabular}{|c|c|c|c|c|c|}
\hline Variables explicativas & Modelo 1 & Modelo 2 & Modelo 3 & Modelo 4 & Modelo 5 \\
\hline \multirow{2}{*}{ Remesas } & $0,252^{* * *}$ & $0,050^{*}$ & $0,051^{* *}$ & $0,091^{*}$ & $0,062^{*}$ \\
\hline & $(0,04)$ & $(0,026)$ & $(0,026)$ & $(0,056)$ & $(0,036)$ \\
\hline \multirow{2}{*}{ Graduados de educación superior } & & $0,50^{* * *}$ & $0,50^{* * *}$ & & \\
\hline & & $(0,05)$ & $(0,05)$ & & \\
\hline \multirow{2}{*}{ Inversión pública } & & $0,575^{* * *}$ & $0,565^{\star * *}$ & & $0,974^{* * *}$ \\
\hline & & $(0,071)$ & $(0,072)$ & & $(0,095)$ \\
\hline \multirow{2}{*}{ Apertura económica } & & $0,551^{* * *}$ & $0,56^{* * *}$ & $0,70^{* *}$ & $0,483^{* *}$ \\
\hline & & $(0,181)$ & $(0,182)$ & $(0,358)$ & $(0,24)$ \\
\hline \multirow{2}{*}{ Remesas * ciclo económico } & & & $0,053^{* *}$ & & \\
\hline & & & $(0,05)$ & & \\
\hline \multirow{2}{*}{ Educación y capacitación superior } & & & & $0,225^{*}$ & $0,147^{* *}$ \\
\hline & & & & $(0,116)$ & $(0,074)$ \\
\hline \multirow{2}{*}{$\begin{array}{l}\text { Transparencia institucional y } \\
\text { administrativa }\end{array}$} & & & & $-0,013$ & 0,054 \\
\hline & & & & $(0,081)$ & $(0,06)$ \\
\hline \multirow{2}{*}{ Infraestructura TIC } & & & & 0,013 & $0,118^{*}$ \\
\hline & & & & $(0,089)$ & $(0,069)$ \\
\hline \multirow{2}{*}{ Dinámica empresarial } & & & & $0,978^{\star * *}$ & $0,485^{\star \star *}$ \\
\hline & & & & $(0,229)$ & $(0,139)$ \\
\hline \multirow{2}{*}{ Constante } & $8,30^{* * *}$ & $-2,94^{* * *}$ & $-2.86^{* * *}$ & $7,80^{* * *}$ & $-4,48^{* * *}$ \\
\hline & $(0,167)$ & $(0,699)$ & $(0,713)$ & $(0,318)$ & $(1,227)$ \\
\hline Observaciones & 160 & 160 & 160 & 80 & 80 \\
\hline Periodo & \multicolumn{3}{|c|}{$2009-2016$} & \multicolumn{2}{|c|}{$2013-2016$} \\
\hline R-cuadrado & 0,97 & 0,98 & 0,98 & 0,97 & 0,97 \\
\hline
\end{tabular}

\section{Pruebas de elección}

\begin{tabular}{|c|c|c|c|c|c|}
\hline Prueba de Breusch-Pagan & $520^{* * *}$ & $395^{* * *}$ & $395^{\star * *}$ & $96^{* * *}$ & $78^{* * *}$ \\
\hline Prueba F & $441^{* \star *}$ & $230^{* * *}$ & $258^{* * *}$ & $1147^{* * *}$ & $398^{* * *}$ \\
\hline Prueba de Hausman & $5^{* \star}$ & $53^{* * *}$ & $61^{\star \star *}$ & $21^{* \star *}$ & $81^{* * *}$ \\
\hline \multicolumn{6}{|l|}{ Probando los supuestos del error } \\
\hline Prueba de heterocedasticidad & $160^{* * *}$ & $228^{* * *}$ & $239^{* * *}$ & $1954^{* * *}$ & $2661^{* * *}$ \\
\hline Prueba de Autocorrelación & $834^{* * *}$ & $92^{* * *}$ & $72^{\star \star \star}$ & $85^{\star * *}$ & $47^{* * *}$ \\
\hline
\end{tabular}

Errores estándar entre paréntesis. Significativo al: $1 \%^{* * *} ; 5 \%{ }^{* *} ; 10 \%$ *

Fuente: cálculos propios con datos del DANE, OLE, DNP, MinHacienda, Banco de la República, DIAN y el IDC. 


\section{CONCLUSIONES}

Los estudios sobre las remesas internacionales y sus impactos han despertado interés en diversos países de América Latina desde hace varias décadas, y Colombia no ha sido ajena a esta cuestión, dados los grandes flujos de dinero que representan para el país. Históricamente, dichas transferencias se incrementaron en gran medida después del gran auge de emigraciones que se presentaron a finales de los años noventa, a causa de la situación económica y social de aquella época en Colombia (Sánchez \& Echeverry, 2014; Urrutia, 2005).

En el caso de esta investigación, como aplicación empírica en las regiones colombianas en el periodo 2009-2016, añadiendo así nueva evidencia, se pudo observar que los principales lugares de origen de los emigrantes colombianos en el exterior corresponden a departamentos como el Valle del Cauca, Cundinamarca-Bogotá y Antioquia, los cuales también reciben los mayores flujos de remesas de trabajadores en el extranjero. Así mismo, los principales países de destino de estos emigrantes corresponden a naciones como Estados Unidos y España, y de estos lugares se reciben los mayores ingresos por remesas.

En lo relacionado con el crecimiento económico, se pudo observar la relación positiva entre ingresos por remesas internacionales y la generación de la producción bruta departamental, de forma tal que por ejemplo las regiones como Valle del Cauca, Cundinamarca-Bogotá y Antioquia son las que han tenido mayores flujos de emigración internacional, generación de remesas y producción interna bruta.

En lo referente a los resultados econométricos, se encontró que efectivamente las remesas tienen un impacto positivo y significativo sobre el crecimiento económico de los departamentos en Colombia, de forma tal que se logra responder la pregunta de investigación y por lo tanto al objetivo propuesto en este artículo, generando además nueva evidencia empírica a la literatura nacional con un periodo de estudio más reciente.

Así mismo, el impacto positivo de las remesas sobre el crecimiento económico puede explicarse vía demanda interna en cuanto al consumo corriente de los hogares, la compra de vivienda, demanda de servicios educativos y de salud, entre otros (Uribe, 2005; Urrutia, 2005; Khoudour, 2007), pero también vía oferta mediante inversiones en negocios productivos, microempresas $\mathrm{y}$ diferentes clases de emprendimientos (Uribe, 2005; Alquinga, 2014; Zheng $\&$ Musteen, 2018). Como futura investigación se propone analizar, sea con estudios probabilísticos o no probabilísticos, qué parte de las remesas se distribuyen en consumo y cuál en negocios o microempresas. 
Finalmente, en el caso de las variables de control, se pudo observar que los efectos marginales esperados se lograron obtener bajo las estimaciones de efectos fijos para datos panel corregidos por autocorrelación y heterocedasticidad, de tal manera que el capital huma- no, las tecnologías de la información y las comunicaciones, el sector público, la apertura económica y la dinámica empresarial, sí influyen positivamente y de manera significativa sobre el crecimiento económico. 


\section{REFERENCIAS}

Acosta, P., Calderón, C., Fajnzylber, P. \& López (2008). What is the Impact of International Remittances on Poverty and Inequality in Latin America? World Development, 36(1), 89-114. https://doi.org/10.1016/j.worlddev.2007.02.016

Alquinga, M. (2014). Las remesas como estrategia de reproducción familiar, y las inversiones en emprendimientos productivos. (Tesis de Maestría). Facultad Latinoamericana de Ciencias Sociales (FLACSO), Ecuador. Recuperado de http:// repositorio.flacsoandes.edu.ec/handle/10469/7719

Awokuse, T. (2003). Is the Export-led Growth Hypothesis Valid for Canada? Canadian Journal of Economics, 36(1), 126-136. Retrieved from http://www.jstor. org/stable/3131917

Barro, R. \& Lee, J. (2013). A New Data Set of Educational Attainment in the World, 1950-2010. Journal of Development Economics, 104, 184-198. https://doi. org/10.1016/j.jdeveco.2012.10.001

Beck, N. (2001). Time-Series-Cross-Section Data: What Have We Learned in the Past Few Years? Annual Review of Political Science, 4(1), 271-293. https://doi. org/10.1146/annurev.polisci.4.1.271

Beck, N. \& Katz, J. N. (1995). What to Do (and not to Do) with Time-Series Cross-Section Data. American Political Science Review, 89(3), 634-647. Retrieved from http://www.jstor.org/stable/2082979

Bohórquez, D. (2015). El impacto de las remesas sobre la educación en Colombia. (Tesis de Pregrado). Universidad Icesi, Santiago de Cali. Recuperado de https:// repository.icesi.edu.co/biblioteca_digital/bitstream/10906/78581/1/TG00992. pdf

Bonilla, L. (2017). Choques externos y remesas internacionales en las regiones de Colombia. Ensayos sobre Política Económica, 35(84), 189-202. https://doi. org/10.1016/j.espe.2017.05.001

Campo, J. \& Mendoza, H. (2018). Gasto público y crecimiento económico: un análisis regional para Colombia, 1984-2012. Lecturas de Economía, (88), 77-108. 
Recuperado de http://www.scielo.org.co/pdf/le/n88/0120-2596-le-88-00077. pdf

Cardona, D., Montenegro, A. \& Hernández, H. (2017). Creación de empresa como pilar para el desarrollo social e integral de la región Caribe en Colombia: apuntes críticos. Revista Saber, Ciencia y Libertad, 12(1), 130-139. https://doi. org/10.18041/2382-3240/saber.2017v12n1.698

Carvajal, L. \& Almonte, L. (2011). Remesas y crecimiento: un análisis estructural para México. Análisis Económico, 26(62). Recuperado de http://www.redalyc. org/pdf/413/41319914011.pdf

Casas, J. A. (2015). Diversificación de las exportaciones y su impacto sobre el crecimiento económico en Colombia. (Tesis de Pregrado). Universidad del Valle, Santiago de Cali. Recuperado de http://bibliotecadigital.univalle.edu.co/bitstre am/10893/8674/1/0508904-P-E-15.pdf

Delfín, F. \& Acosta, M. (2016). Importancia y análisis del desarrollo empresarial. Revista Pensamiento y Gestión, (40), 184-202. http://dx.doi.org/10.14482/ pege. 40.8810

García, G. \& López, R. (2012). Socio-Economic Impact of Broadband in LAC Countries. Working Paper, Inter-American Development Bank. Retrieved from http://kind.wp.tem-tsp.eu/files/2012/06/26.pdf

Hernández, E. \& Raffo, L. (2016). Comercio intraindustrial y costos de ajuste para la industria del Valle del Cauca (1975-2014). Revista Semestre Económico, 19(41), 137-166. Recuperado de https://revistas.udem.edu.co/index.php/economico/ article/view/1963

Khoudour, D. (2007). Migraciones internacionales y desarrollo: el impacto socioeconómico de las remesas en Colombia. Revista de la CEPAL, (92), 143-161. Recuperado de https://core.ac.uk/download/pdf/38673673.pdf

Krugman, P., Obstfeld, M. \& Melitz, M. (2012). Economía Internacional (9 ed.). Madrid: Pearson.

Krugman, P. \& Wells, R. (2016). Macroeconomía (3 ed.). Barcelona: Reverte. 
Leal, Z. (2008). Las remesas y su relación con el crecimiento económico, el consumo y la inversión: el caso de Colombia. Documento de trabajo, Instituto de Estudios del Caribe de la Universidad de Puerto Rico. Recuperado de http:// lanic.utexas.edu/project/etext/llilas/ilassa/2008/leal.pdf

Lucas, R. (1988). On the Mechanics of Economic Development. Journal of Monetary Economics, 22(1), 3-42. https://doi.org/10.1016/0304-3932(88)90168-7

Mankiw, G., Romer, D. \& Weil, D. (1992). A Contribution to the Empirics of Economic Growth. The Quarterly Journal of Economics, 107(2), 407-437. Retrieved from http://www.jstor.org/stable/2118477

Marín, M., Hernández, E. \& Burbano, E. (2018). Impactos del capital humano en las organizaciones empresariales y en los individuos. En Ponencia en el Encuentro Internacional de Investigadores en Administración 2018, Universidad del Valle, Santiago de Cali, Colombia. Recuperado de https://www.academia. edu/38441141/Impactos_del_Capital_Humano_en_las_Organizaciones_Empresariales_y_en_los_Individuos

Mayoral, F. \& Proaño, M. (2015). El impacto de las remesas en el crecimiento económico de América Latina, 1975-2012. América Latina Hoy, (69). Recuperado de http://dx.doi.org/10.14201/alh201569141161

McAuliffe, M. \& Ruhs, M. (2018). Informe sobre las migraciones en el mundo, 2018. Organización Internacional para las Migraciones. Recuperado de https://publications.iom.int/system/files/pdf/wmr_2018_sp.pdf

Mendoza, J. \& Calderón, C. (2006). Impactos regionales de las remesas en el crecimiento económico de México. Papeles de Población, 12(50), 197-221. Recuperado de http://www.redalyc.org/articulo.oa?id=11205012

Monroy, A. (2010). Impacto de las remesas sobre el crecimiento económico regional Colombiano 1994-2007. (Tesis de Pregrado). Universidad de la Salle, Bogotá. Recuperado de http://repository.lasalle.edu.co/bitstream/handle/10185/12422/ T10.10\%20M757i.pdf?sequence $=1 \&$ isAllowed $=\mathrm{y}$

Montoya, D., López, C., Arboleda, P., Ramírez, A. \& Viana, A. (2010). Remesas como instrumento de crecimiento económico en Colombia: remesas y el sector 
de la construcción en Colombia. Negocios Internacionales, 3(2), 64-86. Recuperado de http://publicaciones.eafit.edu.co/index.php/rni/article/view/51

Ochoa, W. (2010). Migración, crecimiento y desarrollo en el Ecuador. Revista Fuente, 1(3), 68-79. Recuperado de http://docplayer.es/18066957-Migracion-crecimiento-y-desarrollo-en-el-ecuador.html

Organización para la Cooperación y el Desarrollo Económicos -OCDE- (2017). Perspectivas de la OCDE sobre la economía digital 2017. Organización para la Cooperación y el Desarrollo Económicos. Recuperado de http://www. oecd.org/governance/perspectivas-de-la-ocde-sobre-la-economia-digital-2017-9789264302211-es.htm

Pavlou, P. (2003). Consumer Acceptance of Electronic Commerce: Integrating Trust and Risk with the Technology Acceptance Model. International Journal of Electronic Commerce, 7(3), 101-134. Retrieved from https://www.jstor.org/ stable $/ 27751067$

Pradhan, G., Upadhyay, M. \& Kamal, U. (2008). Remittances and Economic Growth in Developing Countries. The European Journal of Development Research, 20(3), 497-506. Retrieved from https://www.tandfonline.com/doi/ $\mathrm{abs} / 10.1080 / 09578810802246285$ ?journalCode $=$ fedr20

Raffo, L., Hernández, E. \& Díaz, V. (2018). The Pacific Alliance and the Potential Effects of a Trans-Pacific Economic Cooperation Agreement without the United States. Revista Finanzas y Política Económica, 10(1), 89-110. http://dx.doi. org/10.14718/revfinanzpolitecon.2018.10.1.3

Ramírez, J. \& Aguas, J. (2015). Escalafón de la competitividad de los departamentos de Colombia 2015. Serie Estudios y Perspectivas, (34). Recuperado de https:// repositorio.cepal.org/bitstream/handle/11362/39627/1/S1501237_es.pdf

Romer, P. (1990). Endogenous Technical Change. Journal of Political Economy, 98(5), part 2, S71-S102. Retrieved from https://www.jstor.org/stable/2937632

Romero, E. \& Salinas, D. (2010). La oferta laboral y el papel de las remesas internacionales: estudio de caso para la zona cafetera colombiana: Área Metropolitana Centro Occidente. Perfil de Coyuntura Económica, (15). Recuperado de http:// www.scielo.org.co/pdf/pece/n15/n15a5.pdf 
Sánchez, D. \& Echeverry, D. (2014). Origen e impacto socioeconómico de las remesas de los trabajadores en Colombia. Revista Civilizar de Empresa y Economía, 5(10), 75-90. https://doi.org/10.22518/2462909X.512

Sannassee, R., Seetanah, B. \& Jugessur, J. (2014). Export-Led Growth Hypothesis: A Meta-Analysis. The Journal of Developing Areas, 48(1), 361-385. Retrieved from http://connection.ebscohost.com/c/articles/94148575/ export-led-growth-hypothesis-meta-analysis

Sinisterra, M. (2005). Migración laboral internacional, remesas y crecimiento económico. Estudios Gerenciales, 21(97), 83-100. Recuperado de http://www. icesi.edu.co/contenido/pdfs/Migracion_Laboral_Internacional.PDF

Solimano, A. \& Allendes, C. (2007). Migraciones internacionales, remesas y el desarrollo económico: la experiencia latinoamericana. Series de la CEPAL, (59). Recuperado de https://www.cepal.org/es/publicaciones/5426-migraciones-internacionales-remesas-desarrollo-economico-la-experiencia

Torres, A. (2008). La relación entre apertura y crecimiento económico: nuevos debates de un viejo asunto. Revista Gestión y Región, (5), 7-36. Recuperado de http://biblioteca.ucp.edu.co/ojs/index.php/gestionyregion/article/view/893

Uribe, J. (2005). Remesas de trabajadores y su impacto económico. Revista del Banco de la República, 78(938), 1-17. Recuperado de http://www.banrep.gov. co/es/node/10497

Urrutia, M. (2005). La importancia de las remesas de trabajadores para el crecimiento económico y su evolución reciente en Colombia. Revista del Banco de la República, 76(910), 5-27. Recuperado de http://www.banrep.gov.co/es/ node/10524

Valencia, M. (2012). El impacto de las remesas internacionales en la participación laboral de los hogares en el área metropolitana de Cali-Yumbo 2007. (Tesis de Pregrado). Universidad del Valle, Santiago de Cali. Recuperado de http:// bibliotecadigital.univalle.edu.co/xmlui/bitstream/handle/10893/3721/CB0463878.pdf?sequence $=4$

Weil, D. (2006). Crecimiento Económico. Madrid: Pearson Educación. 
Woodruff, C. \& Zenteno, R. (2001). Remittances and Microenterprises in México. Escuela de Posgrado de Relaciones Internacionales y Estudios del Pacífico, Universidad de California de San Diego (UCSD), Working paper. Recuperado de https://ssrn.com/abstract=282019

Zheng, C. \& Musteen, M. (2018). The Impact of Remittances on Opportunity-Based and Necessity-Based Entrepreneurial Activities. Academy of Entrepreneurship Journal, 24(3), 1-13. Retrieved from https://www.abacademies.org/journals/ academy-of-entrepreneurship-journal-current-issue.html 
